\title{
Le motif de la forêt dans l'album jeunesse : une approche sensible du paysage au travers d'une coloration narrative.
}

\author{
The pattern of the forest in children's books illustration: a sensitive \\ approach to the landscape through the narrative and colour.
}

\author{
Camille Landreau ${ }^{1}$ \\ ${ }^{1}$ Doctorante, LARA-SEPPIA, Université Toulouse - Jean Jaurès (camille.landreau@univ-tlse2.fr)
}

\begin{abstract}
RÉSUMÉ. L'atelier, espace intime de l'artiste, reflète un cheminement de création poïétique. Il est un lieu propice à la recherche, à l'expérimentation sensible afin de développer une pratique plastique singulière. Ainsi je perçois l'atelier comme un écosystème où se crée une interaction entre un être (le designer plasticien), un phénomène (la création) et un objet d'étude in visu (le motif de la forêt dans l'album jeunesse). À travers l'étude de la forêt - qui a une importance particulière dans le conte, puisqu'elle est le lieu où se déroule de nombreuses péripéties issues du schéma narratif et la conception d'ateliers pédagogiques et artistiques autour de l'écologie en école primaire, je désire refléter la démarche de pensée (interprétation) et d'invention (illustration) d'un paysage graphique et plastique (coloration).

ABSTRACT. The workshop, the artist's intimate space, reflects a poetic creation journey. It's a place conducive to research, to sensitive experimentation in order to develop a singular plastic practice. I perceive the workshop as an ecosystem where an interaction is created between a being (the plastic designer), a phenomenon (the creation) and an object of study (the pattern of the forest in the children's books). Through the study of the forest, which has a particular importance in the tale ; since it's the place where many adventures from the narrative scheme take place and the design of pedagogical and artistic workshops around ecology in primary school ; I want to reflect the thought process (interpretation) and invention (illustration) of a graphic and plastic landscape (coloring).

MOTS-CLÉS. Poïétique, Illustration, Coloration, Motif, Projet en Design, Expérience, Conte, Album jeunesse.

KEYWORDS. Poietic, Illustration, Coloration, Pattern, Design project, Experience, Tale, Children's book.
\end{abstract}

\section{Introduction}

À travers ma recherche, ma méthodologie de travail en atelier, il s'agit de percevoir et de comprendre le paysage par une pratique plastique sous le prisme de la couleur et du motif illustré. Puis d'éveiller les consciences autour de l'écologie par la pratique d'ateliers pédagogiques et artistiques en école primaire. Il me paraît opportun de développer le discours d'une illustratrice coloriste engagée dans l'analyse et l'étude de thématiques importantes de nos jours telles que l'écologie et le paysage. Articulant recherche et création, recherches sur la création et en situation de création, la réflexion menée tout au long de ma thèse, Poïétique de la coloration: De la récolte en design à l'expérience créatrice du motif de l'illustration, se met en place dans une dialogique entre recherche - qui se traduit par la récolte d'éléments hétéroclites plastiques, expérimentation - où il est question de l'élaboration d'expériences chromatiques en atelier-laboratoire et projet - tourné vers la mise en coloration de l'illustration. Le fil conducteur est ainsi l'instauration et le questionnement de méthodes de travail, d'offrir des expériences à poétiser pour reconsidérer la forme même de la modélisation. Le développement de processus exploratoires permet ainsi une réflexion entre pensée théorique et pratique artistique. 


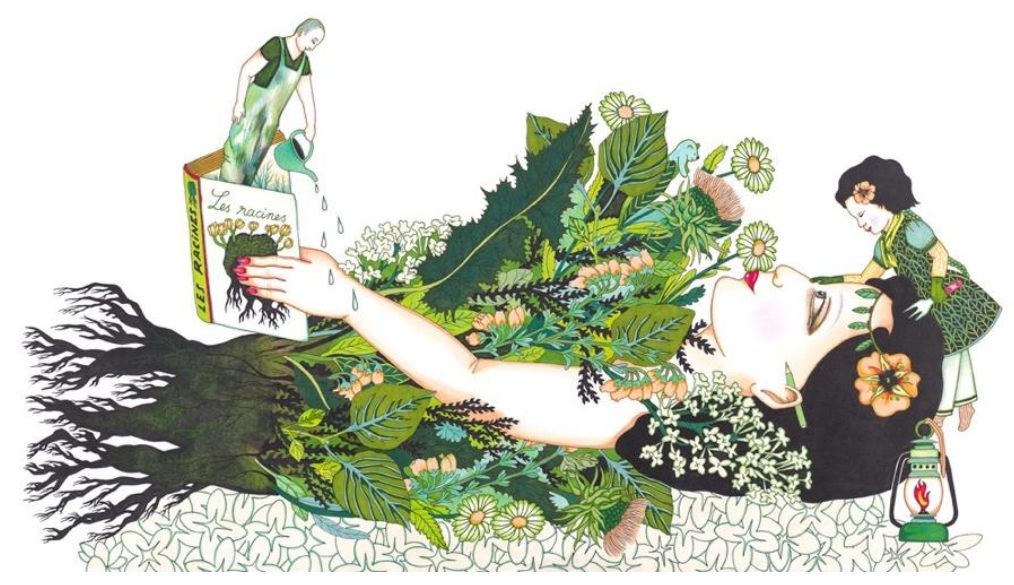

Figure 1. HOUDART Emmanuelle, Ma mère (2015).

\section{L’atelier du designer plasticien : un écosystème poïétique, reflet du processus créatif}

«L'artiste est entouré de multiples environnements, qu'ils soient familiaux, socio-culturels, économiques, matériels ou géographiques, qui encadrent et influencent son œuvre, mais il y a aussi l'environnement que l'artiste se crée lui-même, c'est celui de l'atelier. »'

L'atelier, espace de fabrique intime de l'artiste, reflète un cheminement de création poïétique. Il est un lieu des possibles, d'une infinie variété, propice à la recherche, à l'expérimentation sensible afin de développer une pratique plastique singulière. L'atelier correspond à la posture artistique de celui qui y travaille.

« De tous les cadres, enveloppes (...) qui enferment et « font » l'œuvre d'art (...) il en est un dont on ne parle jamais, que l'on questionne encore moins et qui pourtant, parmi tout ceux qui encerclent et conditionnent l'art, est le tout premier, je veux dire : l'atelier de l'artiste. $»^{2}$

Ainsi je perçois l'atelier comme un écosystème où se crée une interaction entre un être (le designer plasticien), un phénomène (la création) et un objet d'étude in visu (le motif de la forêt dans l'album jeunesse). A travers l'étude de la forêt - qui a une importance particulière dans le conte, puisqu'elle est le lieu où se déroule de nombreuses péripéties issues du schéma narratif - et la conception d'ateliers pédagogiques et artistiques autour de l'écologie en école primaire, je désire refléter la démarche de pensée (interprétation) et d'invention (illustration) d'un paysage graphique et plastique (coloration) pour affirmer le style en devenir de l'illustration. Nous verrons ainsi une vision de la forêt dont la diversité graphique (des motifs et des couleurs) peut évoquer la richesse de la biodiversité.

\section{L’illustration jeunesse : incitation à l'imaginaire}

« Dans le livre, l'image illustre une scène du récit dans un espace limité à la page, tandis qu'elle se libère dans l'album, envahissant le texte et le concurrençant dans ses fonctions narratives et didactiques. Elle ne se contente plus d'illustrer, elle complète, précise, explique, ou apporte un contrepoint. $»^{3}$

\footnotetext{
${ }^{1}$ KORFF-SAUSSE Simone, L'atelier de l'artiste, n¹99, Editions Cazaubon, Le Carnet PSY, p.23-27, 2016/5.

2 BUREN Daniel, Fonction de l'atelier, 1971 in Ecrits vol.1, Bordeaux, CAPC-Musée d'art contemporain, 1991, p195-205.

${ }^{3}$ BNF Classes, L'album : emblème de l'évolution du livre pour enfants, Paris, BNF.
} 
Je suis sensible à l'infinie palette qu'est l'album jeunesse par sa multiplicité des formes, des motifs, des dessins et des couleurs. L'illustration dans l'album jeunesse est devenue un champ de création d'une extraordinaire richesse avec toutes ses variations graphiques. L'album entraîne le lecteur dans un monde imaginaire tout en lui faisant découvrir le pouvoir des mots et des images. Il devient un outil d'enrichissement culturel et un instrument pédagogique.

Dans mon travail de chercheuse plasticienne, je me questionne sur cette manière d'appréhender différemment l'image et la narration du conte, ancré dans notre conscience collective, par la mise en couleur du trait.

Pour ce faire, je mets en place une méthodologie singulière en atelier-laboratoire qui m'amène à analyser des images, des champs lexicaux, expérimenter des couleurs, les classer à travers des cartographies, concevoir des planches d'ambiances, créer des motifs narratifs et un lexique chromatique de l'album jeunesse. Je développe, aiguise et étoffe mon regard sur ce terrain instaurant ainsi une éducation visuelle.

Cela prend tout son sens dans la définition du terme illustrer : « rendre plus clair, plus intelligible, mais aussi donner de l'éclat. » ${ }^{4}$

\section{Une pensée de la couleur au regard du dessin : le motif}

Le dessin a un caractère figuratif, il est l'expression d'une idée. La couleur quant à elle apporte un sens figural, elle est l'apparition de l'abstrait. La couleur est prise sous ses deux significations renouant avec l'étymologie latine du motus qui est d'une part la mise en mouvement et d'autre part le motif déclaré comme motif narratif. C'est dans ce mouvement autour du motif que la narration est susceptible de s'inventer. La couleur peut ainsi se libérer de l'espace du trait pour n'être plus qu'elle-même et devenir couleur-matière. La problématique de fond qui est posée est une dialectique entre le scénario couleur et le processus narratif. La couleur n'est plus un simple outil. La couleur devient une substance actrice dans la création et retranscrit une liberté artistique. L'illustration comme passage et médium pour traduire le dessein à l'image et la coloration comme intention, expérience et narration dans le motif de l'illustration.

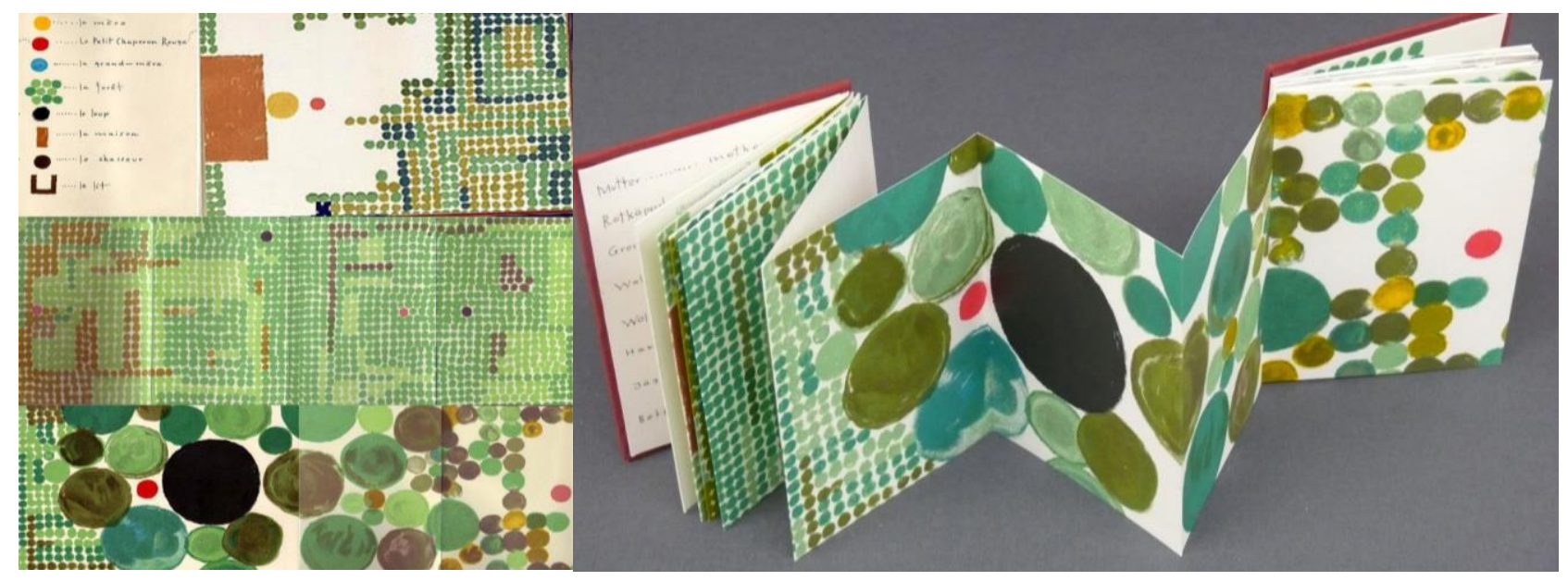

Figurine 2. LAVATER Warja, Le Petit Chaperon Rouge (1965).

\footnotetext{
${ }^{4}$ SOURIAU Etienne, Vocabulaire d'esthétique, Paris, Presses Universitaires de France, PUF, 3ème édition, 2010, (Coll. Quadridge.

Dicos Poche), p.902
} 
«Que l'image devienne une écriture et que l'écriture devienne image. »(LAVATER Warja).

On observe la mise en espace du conte du Petit Chaperon Rouge par la couleur verte sous forme de pictogrammes qui envahissent le support et traduisent le motif de la forêt. Cette dimension ludique et poétique propose une réécriture au moyen de codes visuels afin d'éveiller nos sens, susciter des émotions et questionner notre sensibilité. Par sa polysensorialité, la couleur est à la fois impression et sensation lorsqu'elle devient mouvement et illustration.

La forêt a une importance particulière dans le conte puisqu'elle est le lieu où se déroulent de nombreuses péripéties issues du schéma narratif. couleurs variées qui retranscrivent une ambiance spécifique à l'histoire. L'exploration approfondie des perceptions chromatiques nous amène ainsi à nous interroger sur le processus créatif en tissant des liens entre le conte, l'illustration, le motif et la couleur dans l'album jeunesse.

LA FORÊT : UN MOTIF TRADITIONNEL

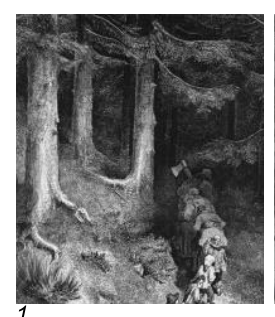

promenade

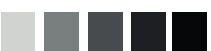

1 - Gustave Doré, Le Petit Poucet, 1867

2 - 3 - Anthony Browne, Dans la forêt profonde, 2004

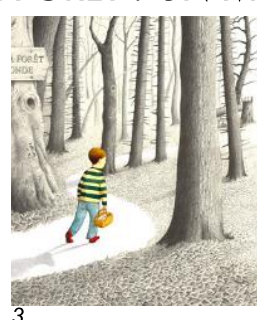

chemin

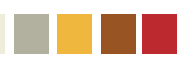

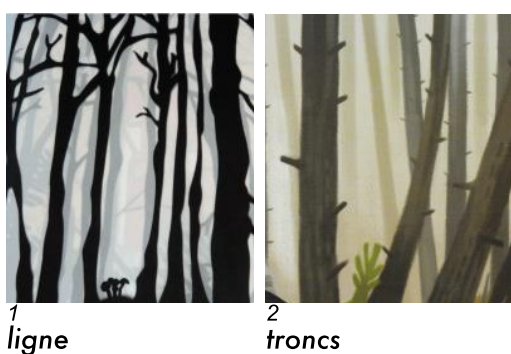

ligne

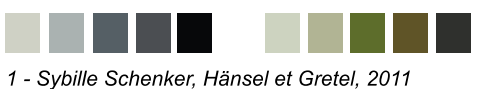

2 - Line Parmentier, Les six cygnes, 2011

3 - Maria Dek, En forêt, 2015

LA FORÊT : UN MOTIF CONTEMPORAIN

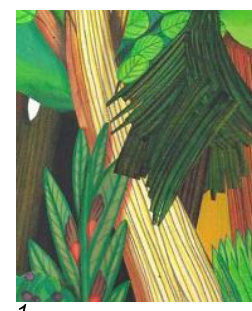

chevauchement

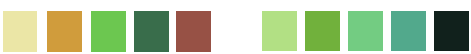

1 - Chiara Dattola, La p'tite forêt, 2014

2 - llya Green, Bou et les 3 zours, 2008 3 - Eva Offredo, Dans la forêt des drôles de bruits, 2017

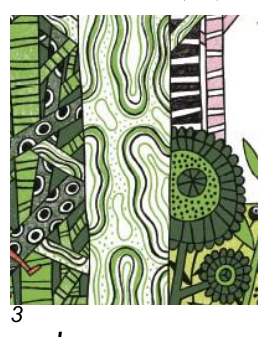

contour

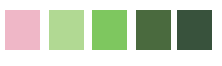

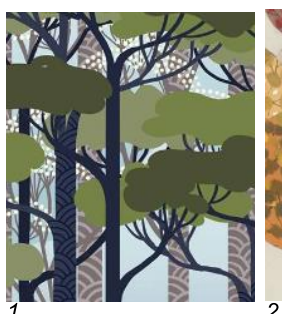

hauteur

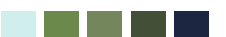

1 - Charlotte Gastaut, Legends \& Farytales, 2016

2 - Nathalie Minne, Le petit garçon de la forêt, 2012

3 - Camille Garoche, La chèvre de Monsieur Seguin, 2014

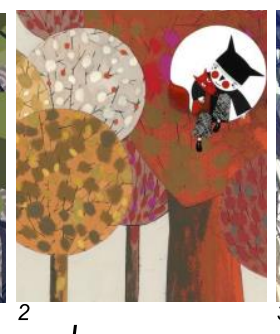

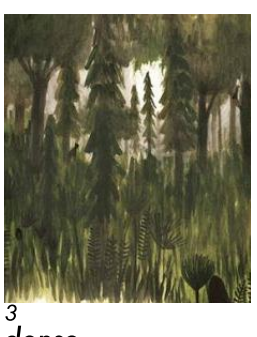

dense
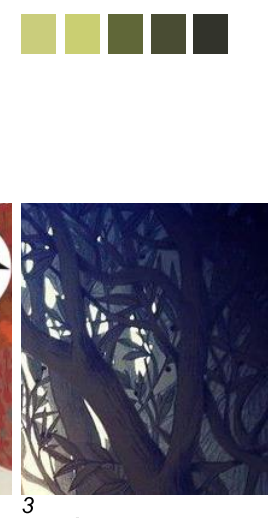

sombre

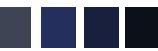

Figure 3. LANDREAU Camille, Motifs illustrés de la forêt issus d'albums jeunesses (2019).

J'associe ces visuels à un champ lexical reflétant la sensation éprouvée au regard du motif et des couleurs. Le motif narratif s'exprime de lui-même ou lorsqu'il est associé à une couleur et un sens lexical devient plus complexe. On se rapporte à une imagerie collective de ce paysage emblématique qu'est la forêt dans le conte.

La forêt traditionnelle que l'on a à l'esprit est parfois réinterprétée. Les codes visuels sont changés par la simplification des formes et l'utilisation de couleurs vives créant ainsi un nouvel imaginaire.

\section{Représenter la forêt en classe primaire : ateliers artistiques et pédagogiques}

J'ai pu réaliser des ateliers artistiques en classe primaire de CP. J'ai souhaité transmettre une pensée de l'écologie à travers une pratique plastique, sous la notion de questionner le monde. Avec 
l'enseignant et moi-même, les enfants ont eu une première approche d'un comportement éthique, un éveil des consciences autour de la nature, de l'écologie et se sentir responsable vis à vis de l'environnement (les enjeux écologiques sur notre monde actuel). Cela s'est traduit par une démarche d'investigation : questionnement, observation, collecte, description. Chacun s'exprimait sur les différentes représentations, les points de vues, les sensations. Des fiches pédagogiques sur l'arbre et la forêt ont été réalisées pour en explorer sa diversité. Puis j'ai utilisé la pratique artistique, une méthodologie et une technique enseignée pour continuer de les sensibiliser à l'écologie. Les enfants ont acquis une éducation à l'art par des connaissances liées à la culture artistique et une éducation par l'art à travers des techniques d'une pratique artistique. J'ai abordé avec les enfants cette thématique de l'écologie et plus particulièrement de la forêt, à travers les motifs, les couleurs, leurs perceptions du paysage, leurs expérimentations. J'ai pu distinguer le processus de création et la pluralité des interprétations à travers une même demande.

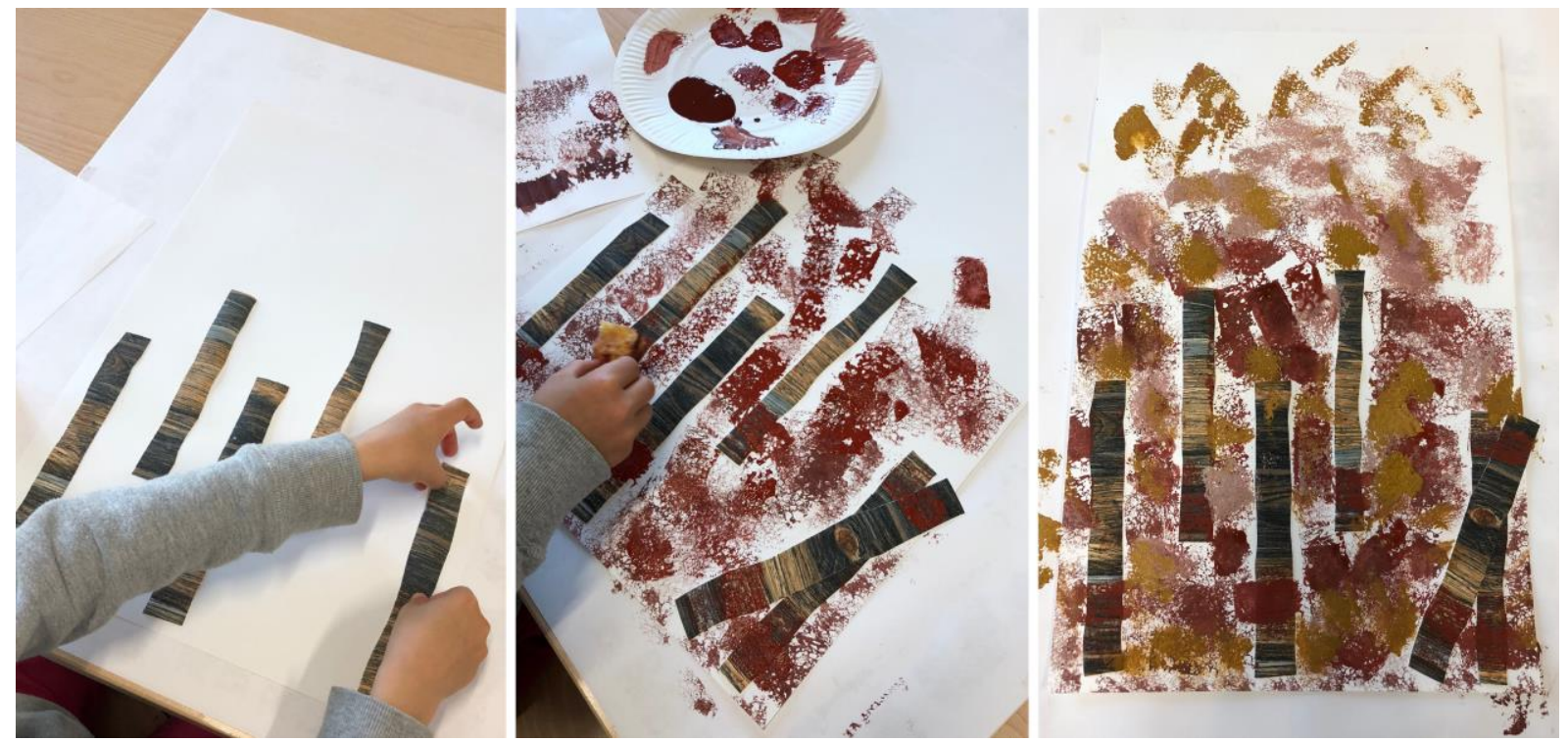

Figure 4. LANDREAU Camille avec les CP de Courbessac, La forêt d'Hansel et Gretel (2018).
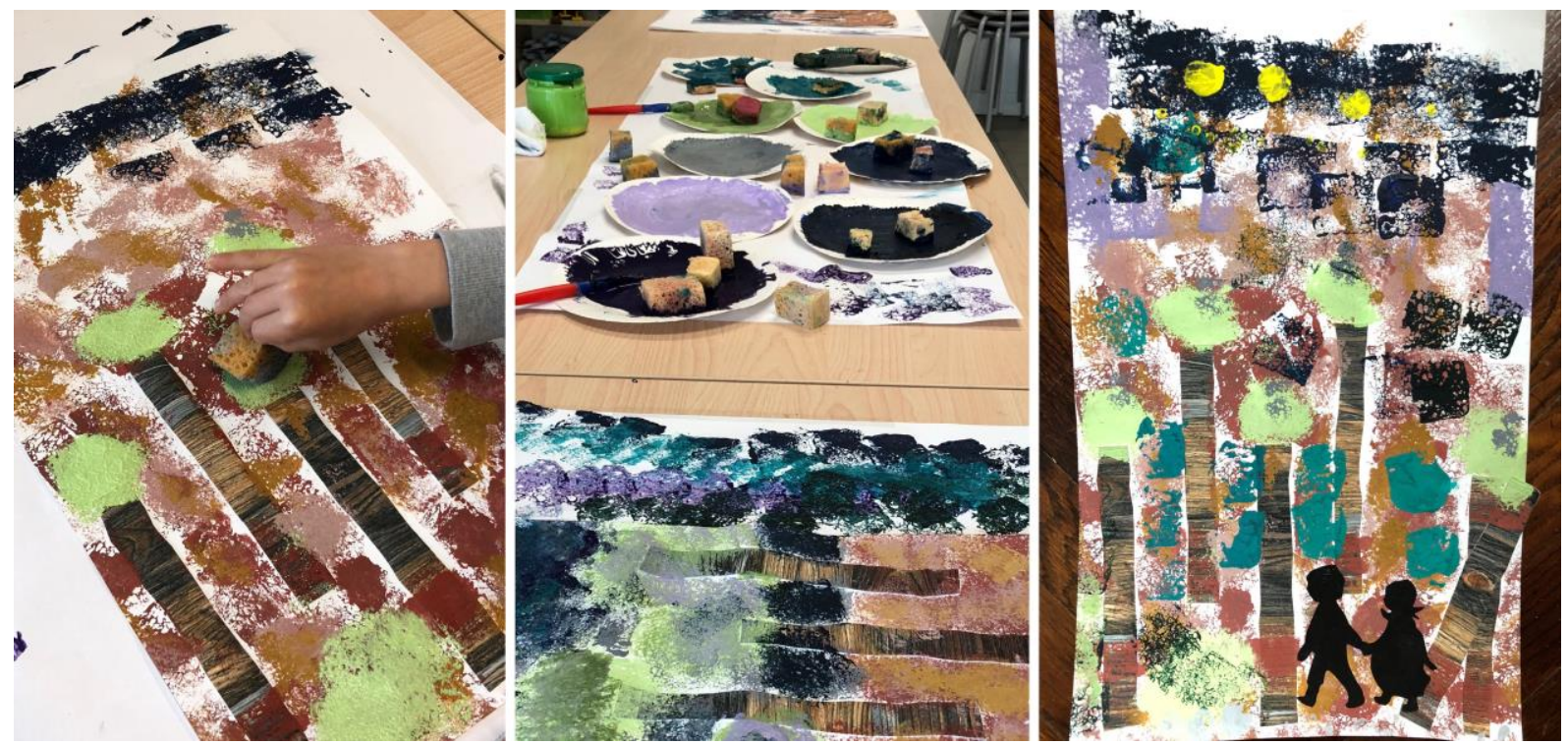

Figure 5. LANDREAU Camille avec les CP de Courbessac, La forêt d'Hansel et Gretel (2018). 


\section{Représentations plastiques : exploration, interprétation et coloration de la forêt}

Il se dégage, à la lecture d'un conte, des lieux magiques, des paysages fantastiques, des personnages énigmatiques, des textures mystérieuses et des atmosphères inquiétantes. Cet univers spécifique permet de créer des variations plastiques à travers l'expérience sensible du vert. Il s'agit, par des planches d'ambiances, de rendre palpable des émotions et des ressentis du récit. La forêt dans une dualité chromatique est tour à tour sombre, hostile et inquiétante puis merveilleuse, accueillante et rassurante.
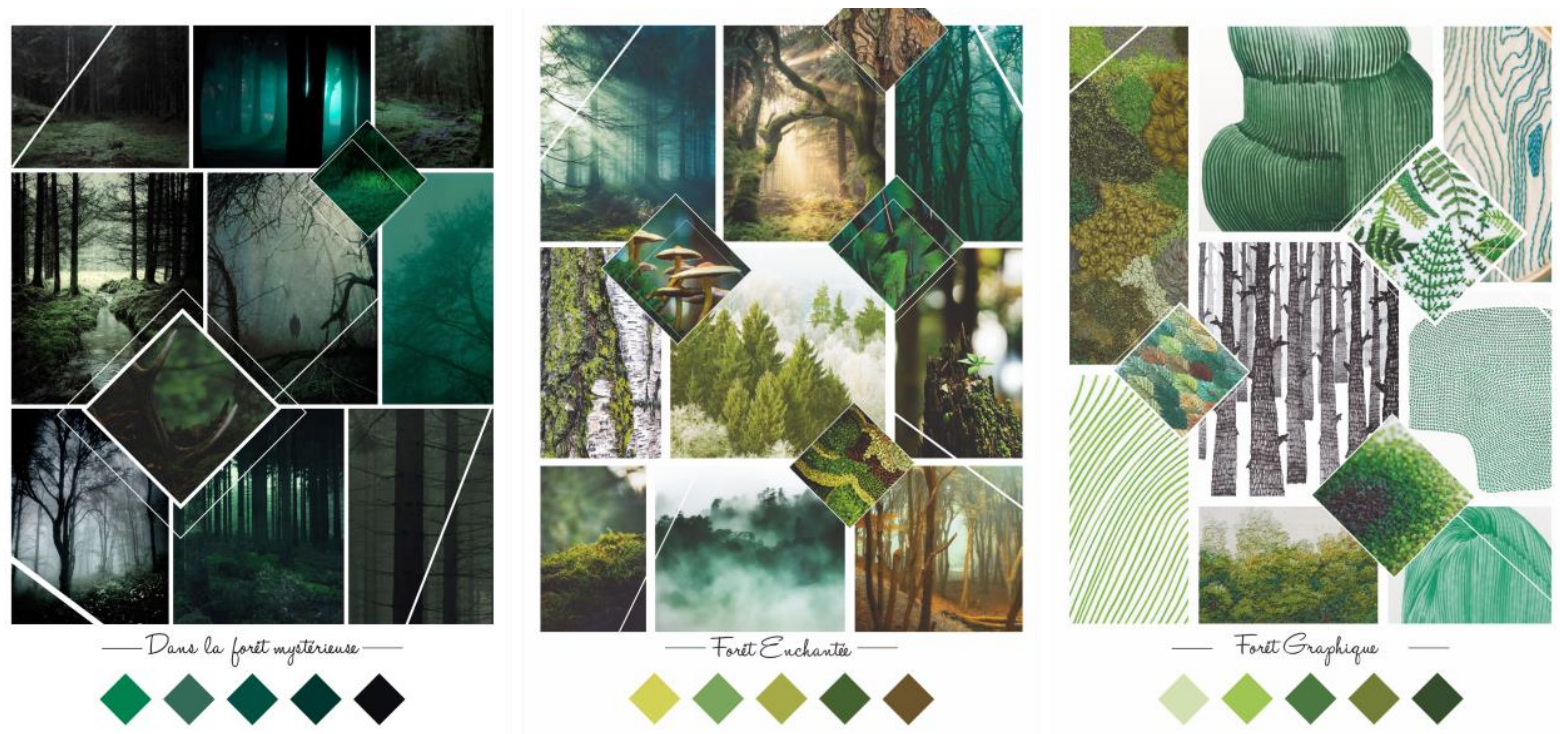

Figure 6. LANDREAU Camille, Planches d'ambiances sur la forêt (2019).

Par la conception d'un répertoire de motifs narratifs, j'interprète l'espace poétique de la forêt afin de concevoir un nouveau langage esthétique.

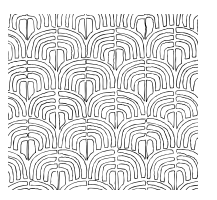

massive

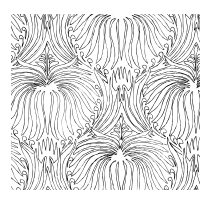

luxuriante

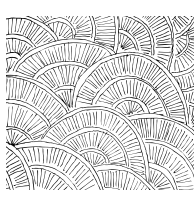

superposition

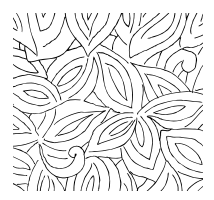

propagation

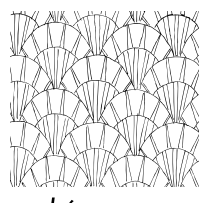

caché

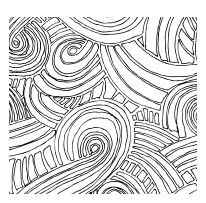

venteux

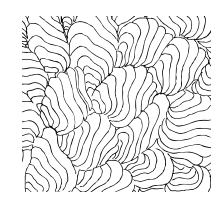

mystère

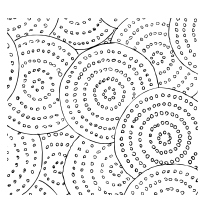

cime

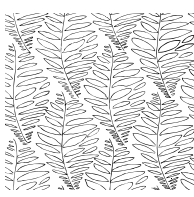

fevillage

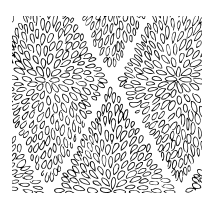

buissons

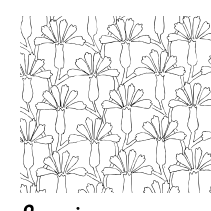

floraison

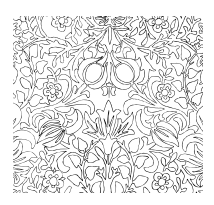

végétation

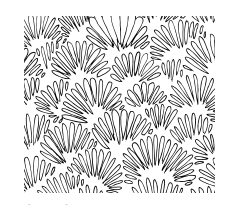

herbes

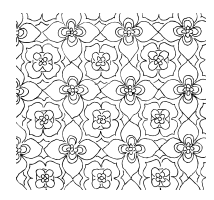

floral

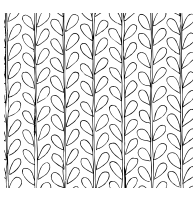

longiligne

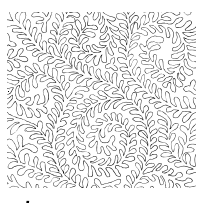

plante

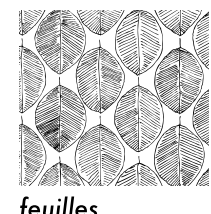

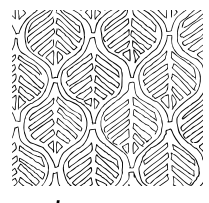

parterre
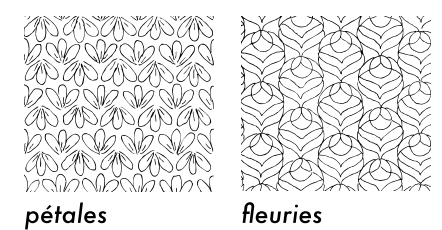

fleuries

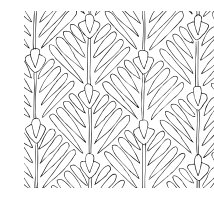

bourgeon

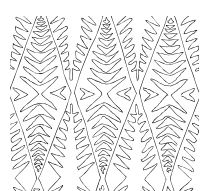

cassant

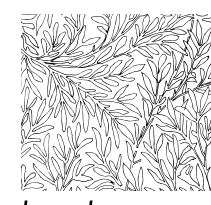

branches

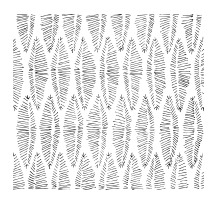

écorce

Figure 7. LANDREAU Camille, Répertoire de motifs La Forêt (2015-2019). 
Ci-dessous, on observe une mes planches autour de la forêt du conte Hansel et Gretel poiétique de la planche : à gauche, la matrice noir et blanc où le motif dessin se compose et à droite, la matrice, le motif et la couleur font coloration.

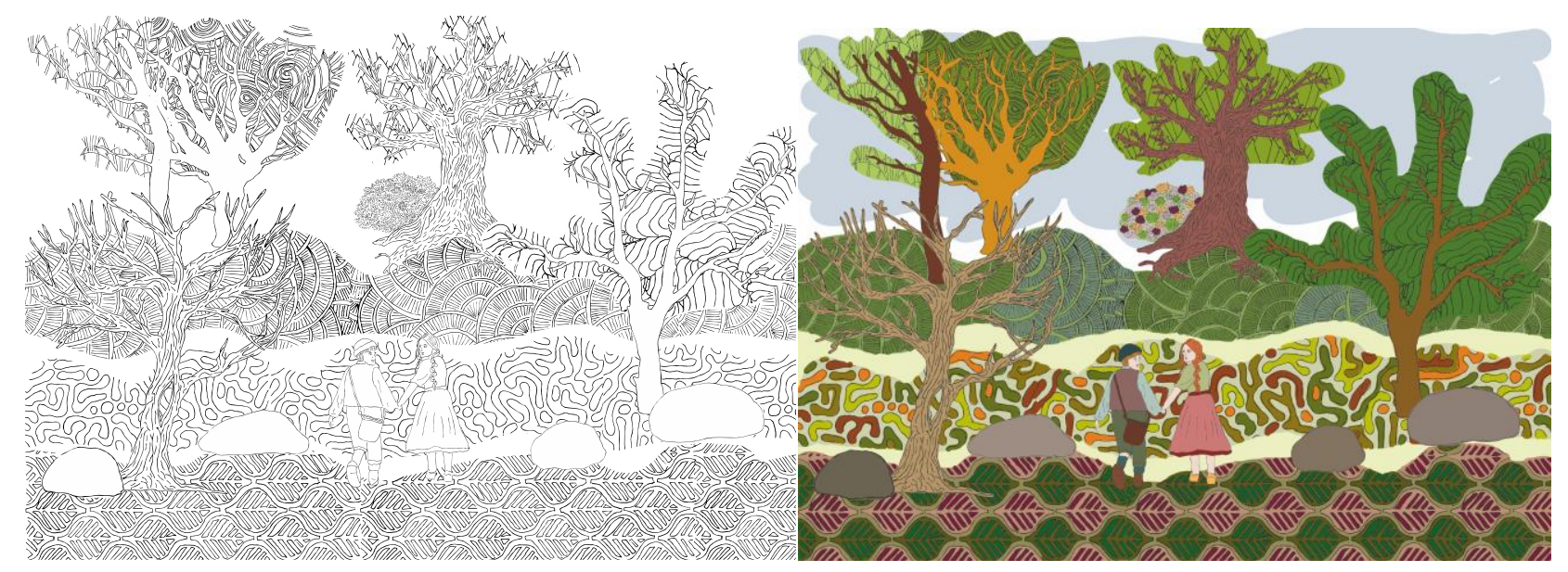

Figure 8. LANDREAU Camille, Planche illustrée et colorée Seuls dans la forêt, (2015).
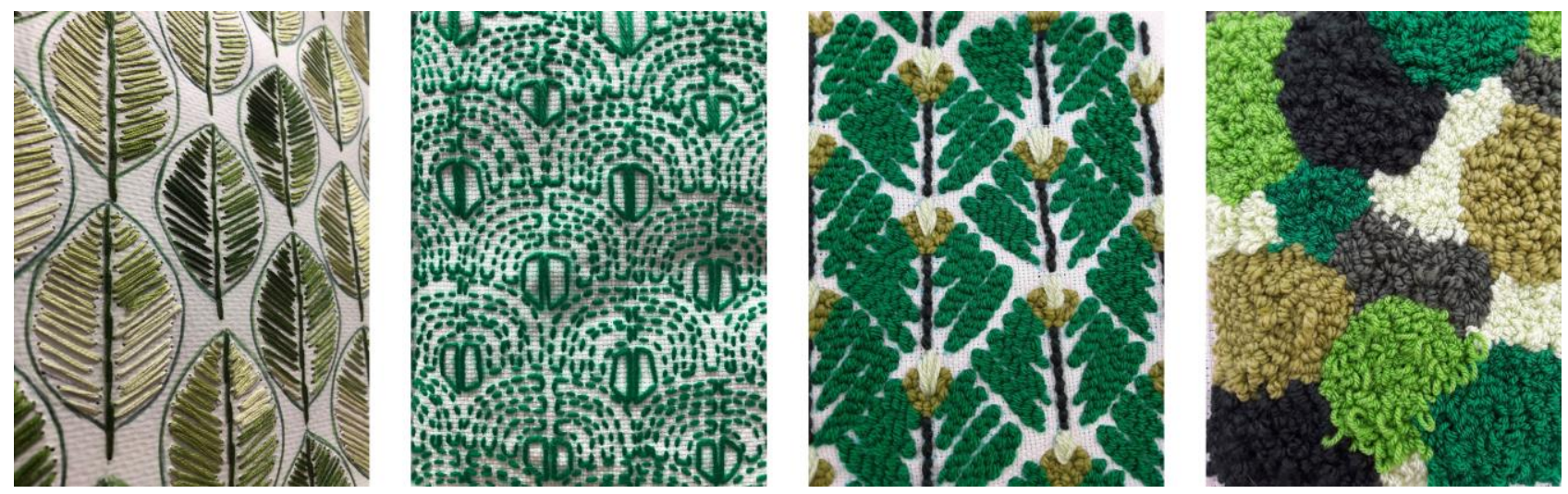

Figure 9. LANDREAU Camille, Motifs brodés (2019).

Par ces tableaux poétiques paysagers, il s'agit de percevoir les multiples interprétations du vert grâce au passage du dessin « le trait illustré » à une expérimentation textile « le fil brodé ». Le graphisme à la fois papier et textile « prend couleur ». En effet, la coloration verte envahit progressivement le médium associé pour révéler ses palettes variées. Chaque perception du vert fait ressortir un imaginaire. Les facettes du dessin et de la broderie dialoguent et s'enrichissent pour donner naissance à un projet plastique singulier et sensible. 

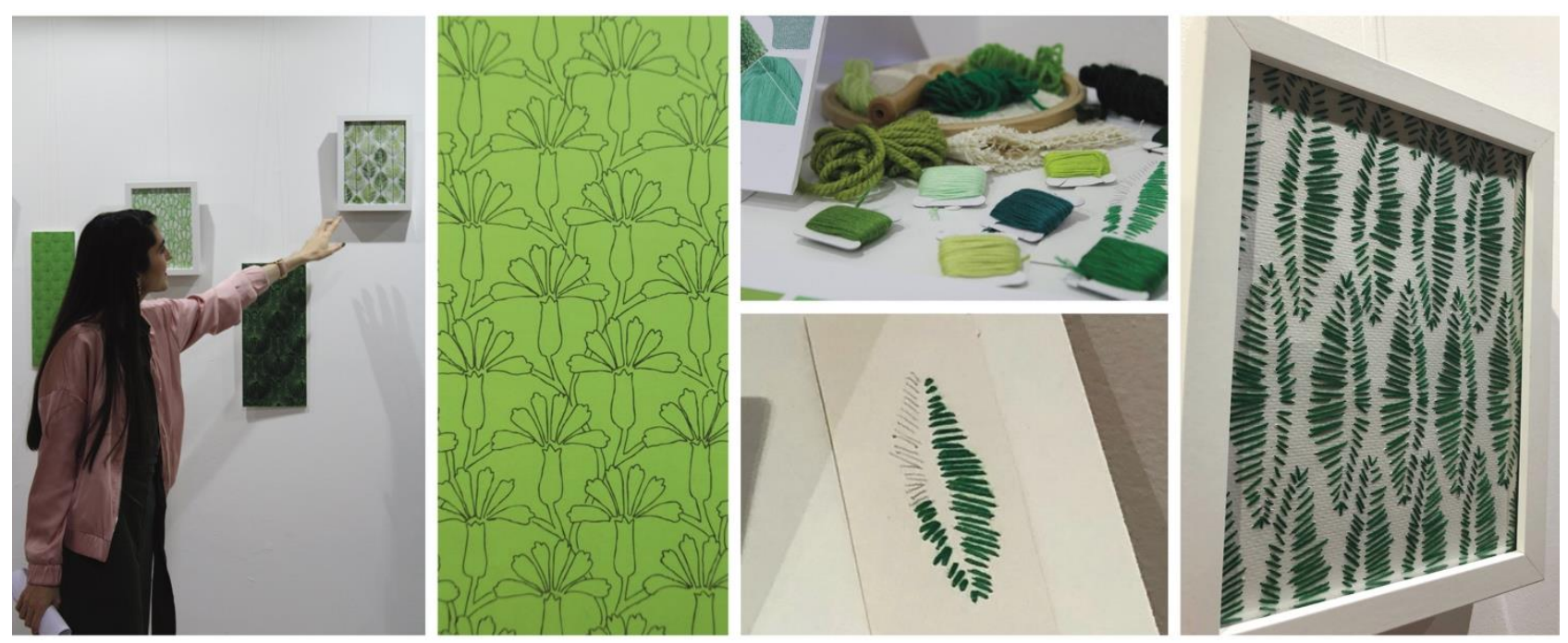

Figure 10. LANDREAU Camille, Du dessin motif à l'expérimentation textile : coloration du vert (2019).

\section{Conclusion}

À travers cette étude autour du motif de la forêt dans l'illustration d'album jeunesse et une pratique artistique personnelle et tournée vers une pédagogie, on perçoit la notion de paysage par le motif narratif qui émane de la construction d'un récit, de la mise en exergue d'une atmosphère. De l'éveil des sensibilités autour de l'écologie par une compréhension d'un lieu in situ et in visu par la pratique artistique. On observe ainsi que la couleur intervient comme une véritable valeur ajoutée. La couleur est un langage qui permet, par une étude méthodique et sensible, de comprendre les enjeux de l'illustration et de leur importance dans notre culture visuelle ainsi que dans leurs enseignements pédagogiques. Cela développe une fabrication du regard, des façons de voir et d'entreprendre ce mode d'expression particulier.

\section{Bibliographie}

ALARY Viviane, L'album : le parti pris des images, Presses Universitaires Blaise Pascal, 2012.

ALBERS Josef, L'interaction des couleurs, Paris, Hazan, 2008.

BNF Classes, L'album : emblème de l'évolution du livre pour enfants, Paris, BNF.

BRUSATIN Manlio, Histoire des couleurs, Paris, Champs-Flammarion, 1986.

BUREN Daniel, Fonction de l'atelier, 1971 in Ecrits vol.1, Bordeaux, CAPC-Musée d'art contemporain, 1991, p195205.

ESCARPIT Denise, La littérature de jeunesse : itinéraires d'hier à aujourd'hui, Paris, Magnard, 2008.

GONDRAND Hélène, Adapter des xuvres littéraires pour les enfants : enjeux et pratiques scolaires, Grenoble, Canopé - CRDP de Grenoble, 2008, (Coll. Les cahiers de Lire écrire à l'école).

KORFF-SAUSSE Simone, L'atelier de l'artiste, n¹99, Editions Cazaubon, Le Carnet PSY, p.23-27, 2016/5.

LORANT-JOLLY Annick, Images des livres pour la jeunesse : lire et analyser, CRDP de Créteil, 2006.

MAJA Daniel, Illustrateur jeunesse : comment créer des images sur les mots ?, Paris, Editions du Sorbier, 2004, (Coll. La littérature de jeunesse).

SOURIAU Etienne, Vocabulaire d'esthétique, Paris, Presses Universitaires de France, PUF, 3ème édition, 2010, (Coll. Quadridge. Dicos Poche).

TORNAY Serge, Voir et nommer les couleurs, Nanterre, Laboratoire d'ethnologie et de sociologie comparative, 1978. VAN DER LINDEN Sophie, Lire l'album, Paris, L'atelier du Poisson soluble, 2006. 